Article

\title{
Optimizing Viscoelastic Properties of Rubber Compounds for Ballistic Applications
}

\author{
Janis Karl ${ }^{1,2, *}$, Franziska Kirsch ${ }^{2}$, Norbert Faderl ${ }^{1}$, Leonhard Perko ${ }^{2}$ and Teresa Fras ${ }^{1}$ (i) \\ 1 French-German Research Institute of Saint-Louis, 5 Rue du Général Cassagnou, 68300 Saint-Louis, France; \\ norbert.faderl@isl.eu (N.F.); teresa.fras@isl.eu (T.F.) \\ 2 Fraunhofer-Institute for Structural Durability and System Reliability LBF, Schlossgartenstr. 6, \\ 64289 Darmstadt, Germany; franziska.kirsch@lbf.fraunhofer.de (F.K.); \\ leonhard.perko@lbf.fraunhofer.de (L.P.) \\ * Correspondence: janis.karl@isl.eu; Tel.: +49-6151-705-8713
}

Received: 1 October 2020; Accepted: 3 November 2020; Published: 5 November 2020

check for updates

\begin{abstract}
Using interlayers of rubber adds a positive effect to the synergy of disruptor-absorber armors. Emerging from its viscoelasticity the material is able to transform mechanical stress into heat. The dynamic mechanical properties of elastomers depend on both ambient temperature and frequency of an applied mechanical load. The damping shows a maximum in the glass transition area. If the frequency of the glass transition is in the magnitude of the mechanical stress rate applied by ballistic impact, the elastomer will undergo the transition and thus show maximized damping. An ideal material for ballistic protection against small calibers is developed by making use of dynamic mechanical analysis and the time-temperature superposition principle. The material is later analyzed by ballistic experiments and compared to other nonideal rubbers with regard to glass transition temperature, hardness and damping. It is shown that by choosing a material correctly with certain glass transition temperature and hardness, the ballistic properties of a steel-rubber-aluminum armor can be enhanced. The chosen material (butyl rubber) with a hardness of $50^{\circ} \mathrm{ShA}$ is able to enhance energy absorption during ballistic impact by around $8 \%$, which is twice as good as other rubber with non-optimized properties.
\end{abstract}

Keywords: multilayered armor; energy absorption; rubber; small-caliber ballistic impact

\section{Introduction}

Due to a steadily increasing threat of sniper attacks, it becomes more important to provide more efficient armor systems not only for the military but also for the protection of civilian infrastructure and vehicles. Modern protection systems tend to use lighter and cost-efficient multilayered armors. An example for this are disruptor-absorber systems interlayered with different materials, instead of classic steel-only armors, which would have to be thick and thus heavy to stop a high-energy kinetic threat. These use interlayer materials ranging from ceramics and metal foams to polymers like polyaramide (Kevlar) or ultra-high-molecular-weight polyethylene (Dyneema) [1-4].

Each part of the multilayered armor plays a specific role. The front-plate is a high-strength and usually brittle layer that shatters incoming projectiles. The back-plate has to be a ductile material that is able to absorb the remaining energy by transforming it into other forms of energy, mostly deformation energy. When a projectile hits a high-strength and usually brittle layer, a compressive stress wave is generated and propagates through the plate. When it reaches the back face of the plate, it is partially reflected back as a tensile wave, which may cause damage and cracks of more brittle materials. The underlying layer should then act as a shock absorber, damp the transmitted impact energy and spread its kinetic energy over a broader area to prevent damage to the first layer $[2,3,5]$. 
A promising material for such an interlayer could be rubber due to its viscoelasticity and high damping properties. Rubber is a composite material consisting of crosslinked polymer and active filler. This enables it to be stretched under mechanical stress while returning to its original shape when the stress is removed. It was reported in 1993 [6] that natural rubber improves the ballistic properties of a steel-rubber-steel armor in comparison to an armor comprising an air gap. Later more effort was put into researching the effect of rubber in laminated armors [6-9].

The phenomenon mainly responsible for the positive effect of rubber applied as an interlayer is thought to be its viscoelasticity, resulting in the ability to damp mechanical stress by transforming it into heat. The viscoelasticity of rubber is defined by its dynamic mechanical properties, such as storage modulus $E^{\prime}$, loss modulus $E^{\prime \prime}$ and loss factor $\tan \delta$. Storage modulus $E^{\prime}$ and loss modulus $E^{\prime \prime}$ are used to describe the elastic and the viscous part of the dynamic modulus of a viscoelastic material and measure the stored and dissipated energy. Loss factor $\tan \delta$ is used to describe the damping ability of the material and is defined as the ratio of $E^{\prime}$ and $E^{\prime \prime}$. These properties are dependent on both the ambient temperature and the frequency of the applied mechanical load. In the range of the glass transition, both loss modulus $E^{\prime \prime}$ and loss factor $\tan \delta$ reach a maximum $[7,10-12]$.

The equivalency of temperature and frequency regarding glass transition can be described by the time-temperature superposition principle. It states that the glass transition can occur in two ways. If the temperature is very low, the thermal energy is not sufficient to overcome the energy barriers of certain polymer chain rearrangements. If the frequency of an applied mechanical stress is very high, the chain segments are not able to react to the applied mechanical load, which is the case for strains close to the resonance frequency of the chain rearrangements. In both cases, the material behaves brittle and glasslike. Hence, temperature and frequency can be used equivalently to describe the glass transition [11].

Bogoslovov et al. [13] reported that by choosing a rubber with a glass transition in the correct frequency range, the ballistic impact induces the glass transition and thus damping is maximized during a ballistic impact. They also reported that the frequency of the shockwave of ballistic impact is around $10^{5} \mathrm{~Hz}$, which according to time-temperature superposition corresponds to a glass transition temperature of around $-50^{\circ} \mathrm{C}$. In a different work from the same group [9], it was examined that by coating steel with a single layer of polyurea, the ballistic limit $v_{50}$ can be improved by $\sim 25 \%$ in comparison to the noncoated steel. Besides the observation of rubber improving ballistic properties, no additional effort was put into further investigating the influence of different material properties.

In this work, improvements of rubber-interlayered armor systems are targeted by selecting an optimized rubber compound for the interlayer with a focus on the influence of damping, hardness and the glass transition temperature on the protective properties of the multilayered armor. An appropriate material is preselected by analyzing the temperature and frequency dependency of the viscoelastic properties using dynamic mechanical analysis (DMA). Furthermore, the selection is validated by different ballistic tests that are used for observing the material response of the rubber layer during the ballistic impact and to determine the energy absorption of the armor configuration. The results are then compared to a reference configuration without rubber and for configurations with different rubbers that are not optimized for the application.

\section{Materials and Methods}

\subsection{Armor Composition}

In the tested armor concept with an elastomer as the interlayer, the armor steel Armox 370T is used as the front, striking-face plate. This steel is a rolled homogenous armor (RHA) grade steel by SSAB (Stockholm, Sweden) that combines excellent shock resistance and toughness. It is characterized by a tensile strength of $1100 \mathrm{MPa}$ and a hardness of $330 \mathrm{HBW}$ [14]. In the tested sandwich composite, the steel plates have a thickness of $6 \mathrm{~mm}$ and dimensions of $25 \mathrm{~cm} \times 25 \mathrm{~cm}$. As the back layer, the aluminum plates (EN AW $5754 \mathrm{HIII}$ ) have a thickness of $4 \mathrm{~mm}$ and the same dimensions. This wrought 
aluminum alloy of magnesium as the main alloying element has a tensile strength of about $200 \mathrm{MPa}$ and an elongation A50 of a minimum of $12 \%$ [15]. Between these two steel and aluminum plates, different rubber compounds are inserted as interlayers.

\subsection{Rubber Compounds}

Rubber compounds are conventional formulations of butyl rubber (X_Butyl RB 301; Arlanxeo, Maastricht, The Netherlands), styrene-butadiene rubber (Buna SBR 2382; Arlanxeo, Maastricht, The Netherlands), butadiene rubber (BUNA cis-132-Schkopau; Trinseo, Berwyn, PA, USA) and natural rubber (SVR CV60; Mai Thao Rubber, Ho Chi Minh City, Vietnam) using commercially available additives. Carbon black (Corax N550; Safic Alcan, Paris, France) is used as a reinforcing filler. Tetramethylenethiuram disulfide (TMTD; Safic Alcan, Paris, France) and mercaptobenzothiazole disulfide (MBTS; Safic Alcan, Paris, France) are used as accelerators for sulfur vulcanization. Zink oxide (Safic Alcan, Paris, France) and Stearic acid (Carl Roth GmbH, Karlsruhe, Germany) are used as activators. Paraffinic oils (Tudalen 3912, Vivatec 200) are obtained from Hansen \& Rosenthal (Hamburg, Germany). The composition of all rubber formulations is listed in Table 1. The rubber-to-metal adhesive system consists of an adhesive primer (Megum 3276; Safic Alcan, Paris, France) and a cover coat (Megum 538; Safic Alcan, Paris, France).

Table 1. Composition of rubber formulations for butyl rubber (IIR), styrene-butadiene rubber (SBR) and butadiene rubber (BR)/natural rubber (NR). (TMTD, tetramethylenethiuram disulfide; MBTS, mercaptobenzothiazole disulfide).

\begin{tabular}{cccc}
\hline Compound & IIR Amount [phr] & SBR Amount [phr] & BR/NR (9:1) Amount [phr] \\
\hline Zinc oxide & 5 & 5 & 5 \\
Stearic acid & 1 & 1 & 1 \\
Carbon black & $20 ; 35 ; 50 ; 65 ; 80^{2}$ & 44 & 45 \\
Paraffinic oil & $10^{2}$ & $20^{3}$ & 10 \\
Sulfur & 1.5 & 1.5 & 1.5 \\
TMTD & 1 & 0 & 0 \\
MBTS & 0.5 & 1.5 & 1.5 \\
\hline
\end{tabular}

Rubber formulations are compounded on an ERMAFA TMI internal mixer (ERMAFA Sondermaschinenund Anlagenbau $\mathrm{GmbH}$, Chemnitz, Germany) using an upside-down process. After putting all additives (carbon black, oil, zinc oxide and stearic acid) into the internal mixer, raw rubber is added. The compound is mixed for $5 \mathrm{~min}$ at a temperature of $40^{\circ} \mathrm{C}$ and a rotor speed of $80 \mathrm{rpm}$. Afterwards, the compound is processed on a Vogt rolling mill to incorporate the crosslinking agents (sulfur, accelerator). Vulcanization is then carried out through compression molding on a Schmidt HB 20300 (Schmidt Maschinentechnik, Bretten-Bauerbach, Germany) laboratory press.

For the production of the specimen used for dynamic mechanical analysis (DMA), between 7 and $9 \mathrm{~g}$ of the raw butyl rubber compound is compression molded for $6 \mathrm{~min}$ at a temperature of $180{ }^{\circ} \mathrm{C}$ and a pressure of 50 bar. The resulting rubber plates have a length and width of $4.5 \mathrm{~cm}$ and a thickness of $2 \mathrm{~mm}$. Specimens with an edge length of $1 \mathrm{~cm}$ get punched out of the rubber plate.

\section{Armor Plates}

To provide proper bonding between all components (steel-rubber-aluminum), the surface of the steel and aluminum plates is sanded to remove oxide layers and then cleaned with acetone. Directly afterwards, the adhesive primer is brushed onto the plates. After a drying time of $30 \mathrm{~min}$, the adhesive cover is applied on top of the primer and the plates are dried for another $30 \mathrm{~min}$.

There are two types of plates produced: rubber-covered aluminum plates and steel-rubber-aluminum sandwich plates. 
Between 480 and $500 \mathrm{~g}$ of the raw rubber compound is placed in a mold between aluminum and steel plates and then vulcanized at a pressure of 150 bar. To ensure that all trapped gases are able to escape the mold, the pressure is reduced again five times before keeping the pressure stable at 150 bar. The vulcanization conditions of each rubber compound necessary to ensure complete vulcanization are listed in Table 2 . These values are obtained by vulcametry.

Table 2. Rubber compounds and vulcanization conditions.

\begin{tabular}{ccc}
\hline Rubber & $\boldsymbol{T}\left[{ }^{\circ} \mathbf{C}\right]$ & $\boldsymbol{t}[\mathbf{m i n}]$ \\
\hline butyl rubber (IIR) & 180 & 30 \\
styrene-butadiene rubber (SBR) & 190 & 30 \\
butadiene rubber (BR)/natural rubber (NR) (9:1) & 180 & 40 \\
\hline
\end{tabular}

\subsection{Dynamic Mechanical Analysis}

The measurements were carried out on a SEIKO SII Exstar 6100 DMS dynamic mechanical analyzer (Seiko Instruments Inc., Chiba, Japan) using tensile mode. Temperature sweeps were measured with a frequency of $1 \mathrm{~Hz}$ in the range of -100 to $0{ }^{\circ} \mathrm{C}$ with a heating rate of $1 \mathrm{~K} / \mathrm{min}$. Frequency sweeps were measured from 0.01 to $100 \mathrm{~Hz}$ at $-50,-40$ and $-30^{\circ} \mathrm{C}$.

\subsection{Ballistic Experiments}

All ballistic experiments were carried out using a powder gun with a distance of $10 \mathrm{~m}$ to the target (Figure 1).

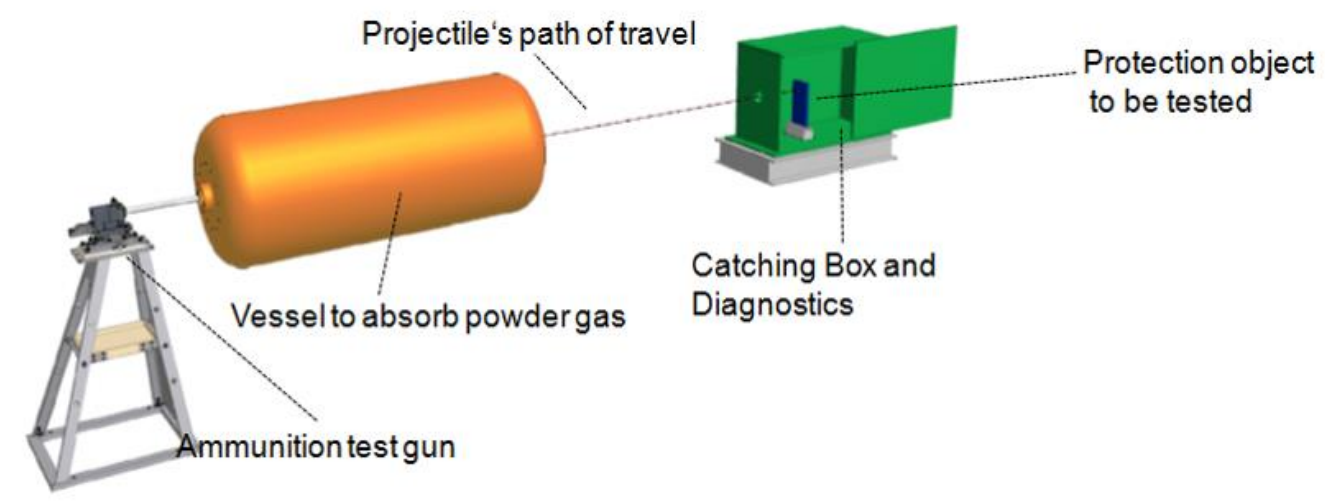

Figure 1. Experimental setup for ballistic experiments [16].

Two types of target configuration were tested under ballistic impact loading. For the determination of the energy absorption, the armor plates consisting of steel-rubber-aluminum were used as a target. As a threat, the small-caliber armor-piercing (AP) projectile M352 $7.62 \times 51$ (Figure 2) with a mass of $4.6 \mathrm{~g}$ was used. The projectile velocity $v_{0}$ was measured using a light barrier. Based on flash X-ray images, the residual velocity $v_{r}$ after target perforation was determined. Each plate was shot two times.

Impact Behavior Observation

To observe the behavior of the rubber layer during impact, the rubber-coated aluminum plates were shot with 7.62 fragment-simulating projectiles (FSP) [17] (Figure 2) and the impact filmed at $250 \mathrm{kfps}$ using an ultra-high-speed camera. In contrast to the armor-piercing 7.62 rounds that have a sharp-pointed nose, the FSPs are characterized by a flat nose. Impacts with this kind of striker allow observations of the behavior of different rubber compounds under an impact. The coated plates were used to better observe the impact response of the rubber interlayer, which would not be as distinctive because of its position between the steel and aluminum plate. 


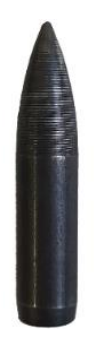

(a)

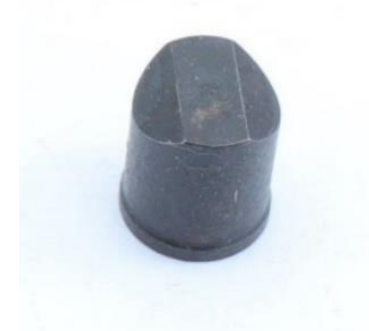

(b)

Figure 2. (a) Projectile M352 $7.62 \times 51$ AP. (b) Projectile 7.62 FSP.

\section{Results}

\subsection{Model for Preselection of Materials}

In order to extrapolate viscoelastic properties of rubber compounds and thus determine the correlation between glass transition frequencies and temperatures, the Williams-Landel-Ferry (WLF) equation [18] can be used:

$$
\log \left(\frac{1}{\beta_{T}}\right)=\frac{-C_{1} \cdot\left(T-T_{0}\right)}{C_{2}+\left(T-T_{0}\right)}
$$

where $\beta_{T}$ is the frequency shift factor, $T_{0}$ is the reference temperature and $C_{1}$ and $C_{2}$ are empirical material and reference temperature-dependent parameters. They describe the influence of the free volume. $C_{1}$ is defined as the reciprocal fractional free volume at the glass transition temperature $T_{g}$, while $C_{2}$ is the ratio of the free fractional volume at $T_{g}$, and $\alpha_{V}$ is the thermal expansion coefficient [19].

The standard procedure to identify glass transition temperatures using a DMA is to carry out a temperature sweep with a frequency of $1 \mathrm{~Hz}$. The glass transition is at the maximum of the $\tan \delta$ peak. The parameter $\beta_{T}$ is the quotient of the peak frequencies at both temperature $T$ and the reference temperature $T_{0}$ :

$$
\beta_{T}=\frac{f_{T}}{f_{0}} .
$$

An example of an extrapolation of a tan $\delta$ curve is given in Figure 3.

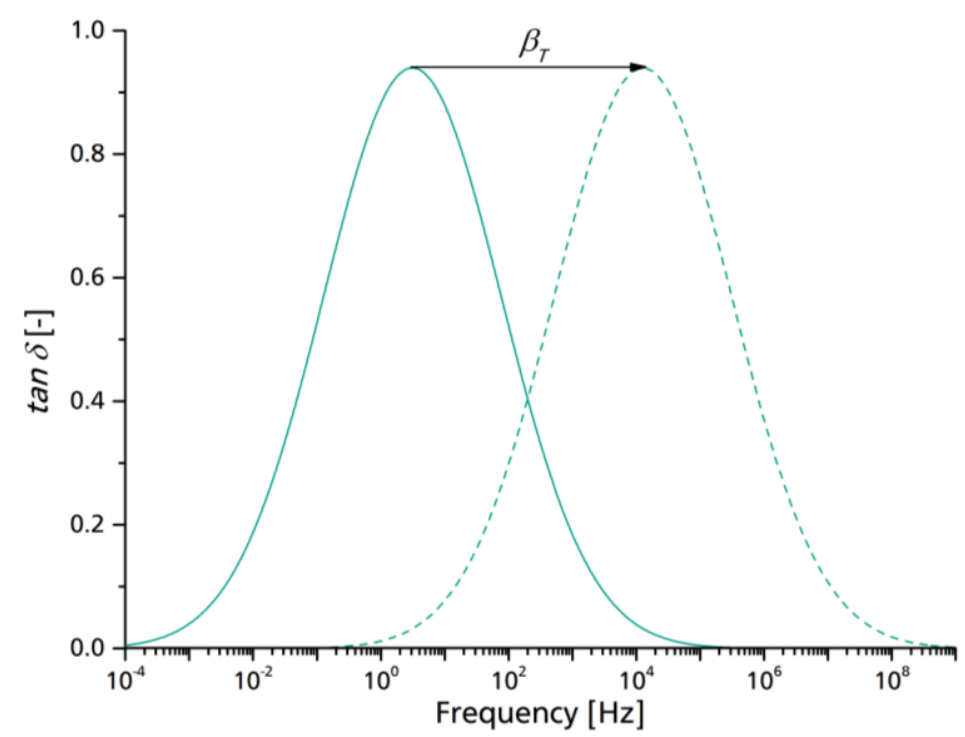

Figure 3. Example of extrapolating a $\tan \delta$ curve to a different temperature using the shift factor $\beta_{T}$. 
If a material for a specific damping application has to be selected, the glass transition frequency at the application temperature needs to be in the range of the load frequency. Referencing the VPAM guidelines [20], common projectiles have impact velocities $v_{0}$ between 300 and $1000 \mathrm{~m} / \mathrm{s}$. Having armor thicknesses $d$ of $5-16 \mathrm{~mm}$ and using

$$
f_{\text {impact }}=\frac{v_{0}}{d}
$$

leads to impact frequencies $f_{\text {impact }}$ ranging from $0.19 \times 10^{5}$ to $2.0 \times 10^{5} \mathrm{~Hz}$. The glass transition frequency $f_{g}$ has to be in that range at room temperature in order to maximize the damping during ballistic impact. According to Equation (2) and using a peak frequency of $1 \mathrm{~Hz}$ at the reference temperature, shift factors range between $0.19 \times 10^{5}$ and $2.0 \times 10^{5}$. Incorporating these shift factors into Equation (1) and using the glass transition temperature $T_{g}$ as $T$ yields an equation that can be used to preselect materials by looking at their glass transition temperature:

$$
T_{g}=\frac{\log \left(\frac{1}{f_{\text {impact }}}\right) \cdot \frac{C_{2}}{C_{1}}}{1+\log \left(\frac{1}{f_{\text {impact }}}\right) \cdot \frac{1}{C_{1}}}-T_{0}
$$

Equation (4) solved with both impact frequencies is plotted in Figure 4.

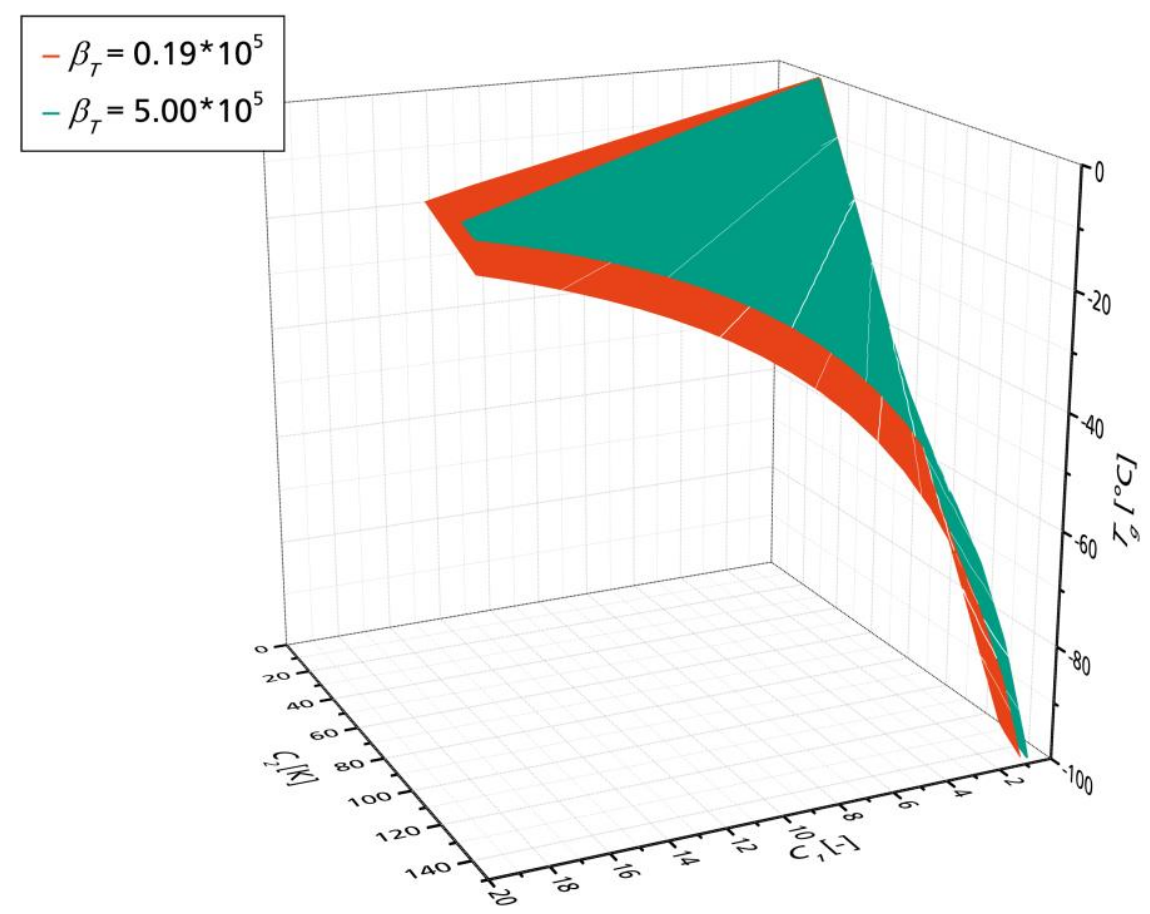

Figure 4. Plots of Equation (4) using $T_{0}=+10^{\circ} \mathrm{C}$ and impact frequencies of $0.19 \times 10^{5}$ and $2.0 \times 10^{5} \mathrm{~Hz}$.

The glass transition temperature of elastomers generally ranges between -100 and $0{ }^{\circ} \mathrm{C}$. By inserting the glass transition temperature of an elastomer into Equation (4), it is possible to calculate the material constants $C_{1}$ and $C_{2}$ that the material needs in order to show maximized damping in the given frequency range. By comparison of these values with those reported in the literature, it is possible to estimate if a material can be used as a damping material as long as the set of all three values $\left(T_{g}, C_{1}\right.$ and $C_{2}$ ) is known. To find out if a material is suitable, it is necessary to mark a point in the diagram that corresponds to the three values of the material. If it is inside the marked range that is bordered by both graphs, then it will have its glass transition frequency in the ideal range. Having a glass transition temperature of $-42{ }^{\circ} \mathrm{C}$, butyl rubber is a promising material with $C_{1}=4.78$ and $C_{2}=81.1 \mathrm{~K}$ at a reference temperature of $T_{0}=+10^{\circ} \mathrm{C}$ [21]. The literature does not mention the exact rubber grade 
and formulation composition, thus these values can only be taken as an indication for the suitability of butyl rubber. To further evaluate this, it is necessary to determine the material properties of a self-manufactured rubber formulation.

\subsection{Time-Temperature Superposition of Butyl Rubber}

In order to set up a model for extrapolating viscoelastic behavior to higher frequencies, a conventional butyl rubber compound was analyzed through dynamic mechanical analysis.

First, a temperature sweep (Figure 5a) was carried out to obtain the glass transition temperature of the rubber compound. Afterwards, frequency sweeps above $T_{g}$ (Figures $5 \mathrm{~b}$ and 6 ) were conducted to characterize the temperature dependence of the glass transition. In both experiments the dynamic moduli $E^{\prime}$ and $E^{\prime \prime}$ were measured for a given temperature or frequency range to examine their temperature and frequency dependency. The peak of $\tan \delta$, which is the ratio of $E^{\prime}$ and $E^{\prime \prime}$, was used to determine the glass transition temperature and frequency.

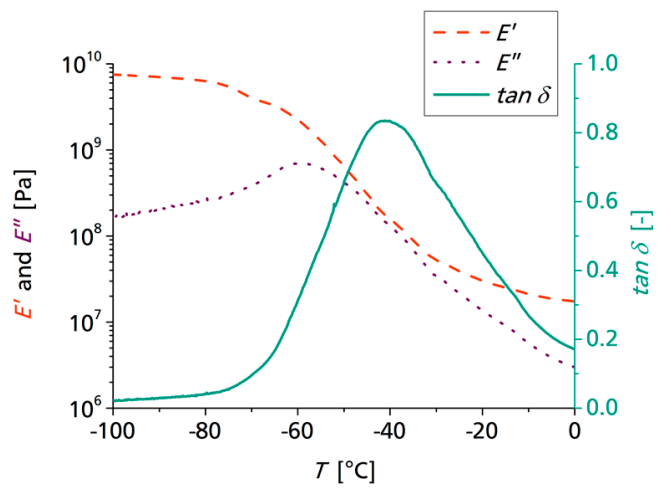

(a)

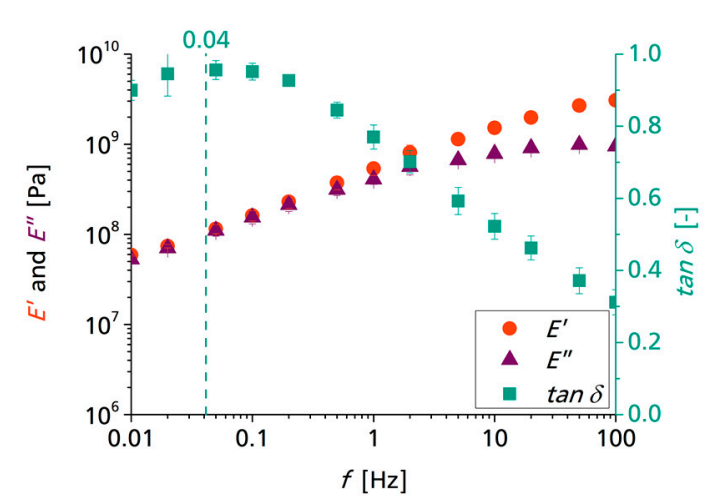

(b)

Figure 5. (a) Temperature sweep ( $1 \mathrm{~Hz}$ ) of butyl rubber from -100 to $-20^{\circ} \mathrm{C}$. (b) Frequency sweep of butyl rubber, $T=-50^{\circ} \mathrm{C}$. Storage modulus $E^{\prime}$, loss modulus $E^{\prime \prime}$ and loss factor tan $\delta$ are plotted against temperature (a) and frequency (b).

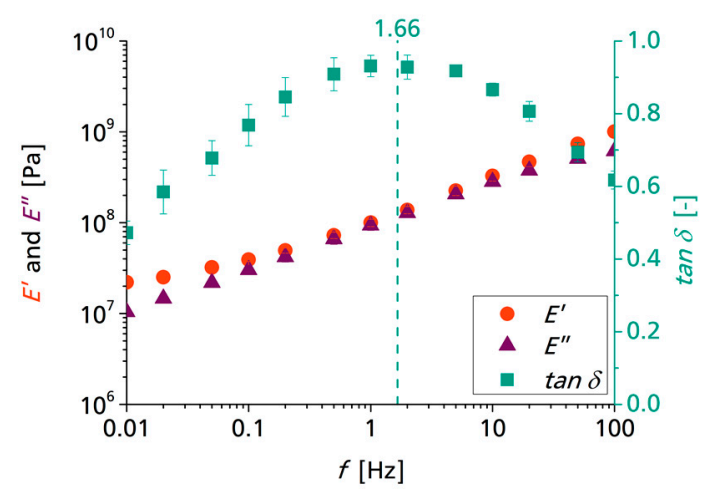

(a)

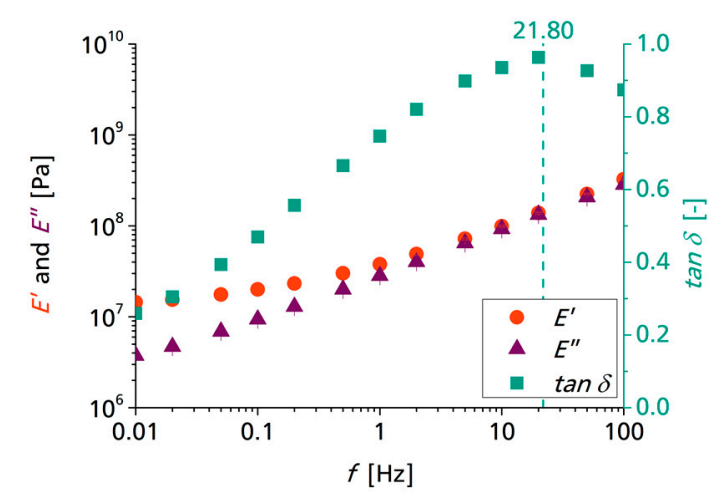

(b)

Figure 6. Frequency sweeps of butyl rubber: (a) $\mathrm{T}=-40^{\circ} \mathrm{C}$ and (b) $\mathrm{T}=-30^{\circ} \mathrm{C}$. Storage modulus $E^{\prime}$, loss modulus $E^{\prime \prime}$ and loss factor tan $\delta$ are plotted against frequency.

According to the time-temperature superposition principle, the shift factors $\beta_{T}$ (Table 3) can be calculated using Equation (2). The temperature of $-50^{\circ} \mathrm{C}$ was chosen as the reference temperature $T_{0}$. As expected, the peak frequency of $\tan \delta$ shifts to higher frequencies for higher temperatures. 
Table 3. Shift factors for reference temperature $T_{0}=-50^{\circ} \mathrm{C}$.

\begin{tabular}{ccc}
\hline$T\left[{ }^{\circ} \mathrm{C}\right]$ & $f_{g}(\tan \delta)[\mathrm{Hz}]$ & $\boldsymbol{\beta}_{T}[-]$ \\
\hline$-50{ }^{\circ} \mathrm{C}$ & 0.04 & 1.00 \\
$-40{ }^{\circ} \mathrm{C}$ & 1.66 & 40.49 \\
$-30{ }^{\circ} \mathrm{C}$ & 21.80 & 531.61 \\
\hline
\end{tabular}

Using the WLF Equation (1), an equation system consisting of two equations for the temperatures -40 and $-30{ }^{\circ} \mathrm{C}$ can be established for determining the material and reference temperature-dependent constants $C_{1}$ and $C_{2}$ :

$$
\Rightarrow C_{1}=8.96 \quad C_{2}=45.73 \quad \mathrm{~K} .
$$

Equation (1) shows that the temperature at which the glass transition occurs at $1 \mathrm{~Hz}$ can be calculated by using the room temperature as the new reference temperature $T^{\prime}$. Keeping in mind that constants $C_{1}$ and $C_{2}$ also depend on the reference temperature, it is necessary to extrapolate the new constants $C_{1}^{\prime}$ and $C_{2}^{\prime}$ for the room temperature by using

$$
C_{2}^{\prime}=C_{2}+\left(T_{0}^{\prime}-T_{0}\right)
$$

and

which lead to

$$
C_{1}^{\prime}=\frac{C_{1} \cdot C_{2}}{C_{2}^{\prime}}
$$

$$
\Rightarrow C_{1}^{\prime}=3.39 \quad C_{2}^{\prime}=120.73 \quad \mathrm{~K} .
$$

It is now possible to determine the temperature range that corresponds to the frequencies induced during ballistic impact. The plot of Equation (1) including the new values for $C^{\prime}{ }_{1}, C^{\prime}{ }_{2}$ and $T^{\prime}$ is shown in Figure 7a. With decreasing armor thickness and increasing projectile velocity, the temperature that corresponds to the impact frequency decreases. By comparison with the temperature sweep of butyl rubber (Figure $7 \mathrm{~b}$ ), it can be concluded that frequencies induced during ballistic impact are located in the area of maximized damping of the material. Even for high projectile velocities $(1000 \mathrm{~m} / \mathrm{s})$ and very thin armors $(5 \mathrm{~mm})$, the temperature of $-49^{\circ} \mathrm{C}$ corresponds to a tan $\delta$ of 0.7 , which still is $84 \%$ of the maximum $\tan \delta$ of the analyzed rubber compound. A $\tan \delta$ of around 0.1 can be seen as the minimum value necessary for a damping material [22]. The experimental values of the manufactured butyl rubber are much higher, which leads to the assumption that it could be a well-suited damping material for ballistic protection.

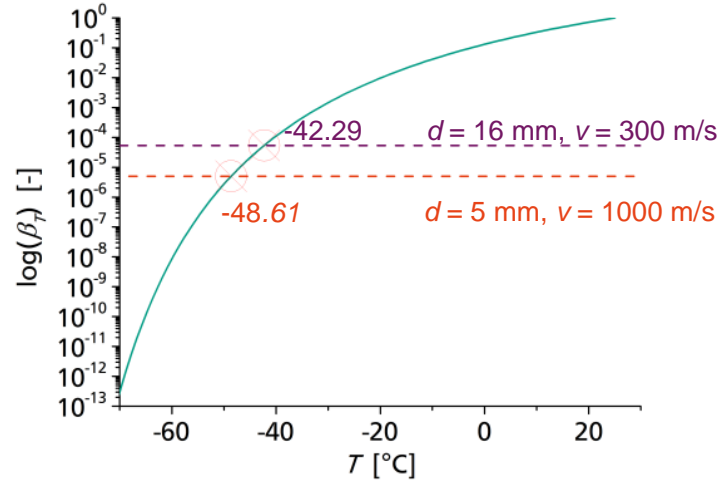

(a)

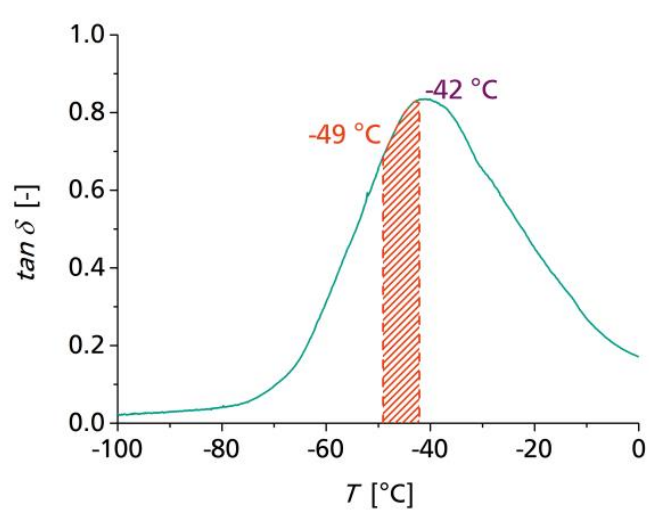

(b)

Figure 7. (a) Plot of the Williams-Landel-Ferry (WLF) equation with $T^{\prime}=25{ }^{\circ} \mathrm{C}, \mathrm{C}^{\prime}{ }_{1}=3.39$ and $C^{\prime}{ }_{2}=120.73 \mathrm{~K}$. (b) Temperature sweep of butyl rubber with the corresponding temperature range for impact-induced frequencies. 


\subsection{Impact Behavior of Rubber-Coated Plates}

In Section 3.2, it is shown that induced frequencies during ballistic impact are located in the area of maximized damping for the butyl rubber compound used, as the material undergoes the glass transition in this frequency range. To provide proof to the claim that this phenomenon positively effects ballistic protection, ballistic experiments were conducted with three different rubber formulations with different glass transition temperatures to cover three cases: an ideal glass transition temperature (in agreement with the results from Section 3.2), a glass transition temperature that is higher than the ideal one, and one that is lower than the ideal one. If an optimized glass transition temperature and thus the maximized damping in this area has a positive effect on ballistic protection, then the rubber compound with the ideal $T_{g}$ should show the best results.

As the influence of fillers and crosslinking system on the glass transition temperature is limited, it has to be varied by choice of different polymers. Butyl rubber (IIR), styrene-butadiene rubber (SBR), and a butadiene rubber (BR)/natural rubber (NR) blend (ratio of 9:1) were used. The elastomers and their respective glass transition temperatures are listed in Table 4. In attempt to reduce other possible influence factors on the results, e.g., hardness, all three formulations were prepared with different carbon black contents to ensure that all three rubber compounds possess the same hardness $\left(\sim 50^{\circ} \mathrm{ShA}\right)$

Table 4. Rubber compounds used for the interlayer in the armored laminates tested.

\begin{tabular}{cc}
\hline Elastomer & $T_{g}\left[{ }^{\circ} \mathrm{C}\right]$ \\
\hline SBR & $-19{ }^{\circ} \mathrm{C}$ \\
IIR & $-42{ }^{\circ} \mathrm{C}$ \\
BR/NR $(9: 1)$ & $-100^{\circ} \mathrm{C}$ \\
\hline
\end{tabular}

In the first experimental series, the impact behavior of the rubber-coated aluminum plates was observed with a high-speed camera. The images of the impact experiments are shown in Figure 8.

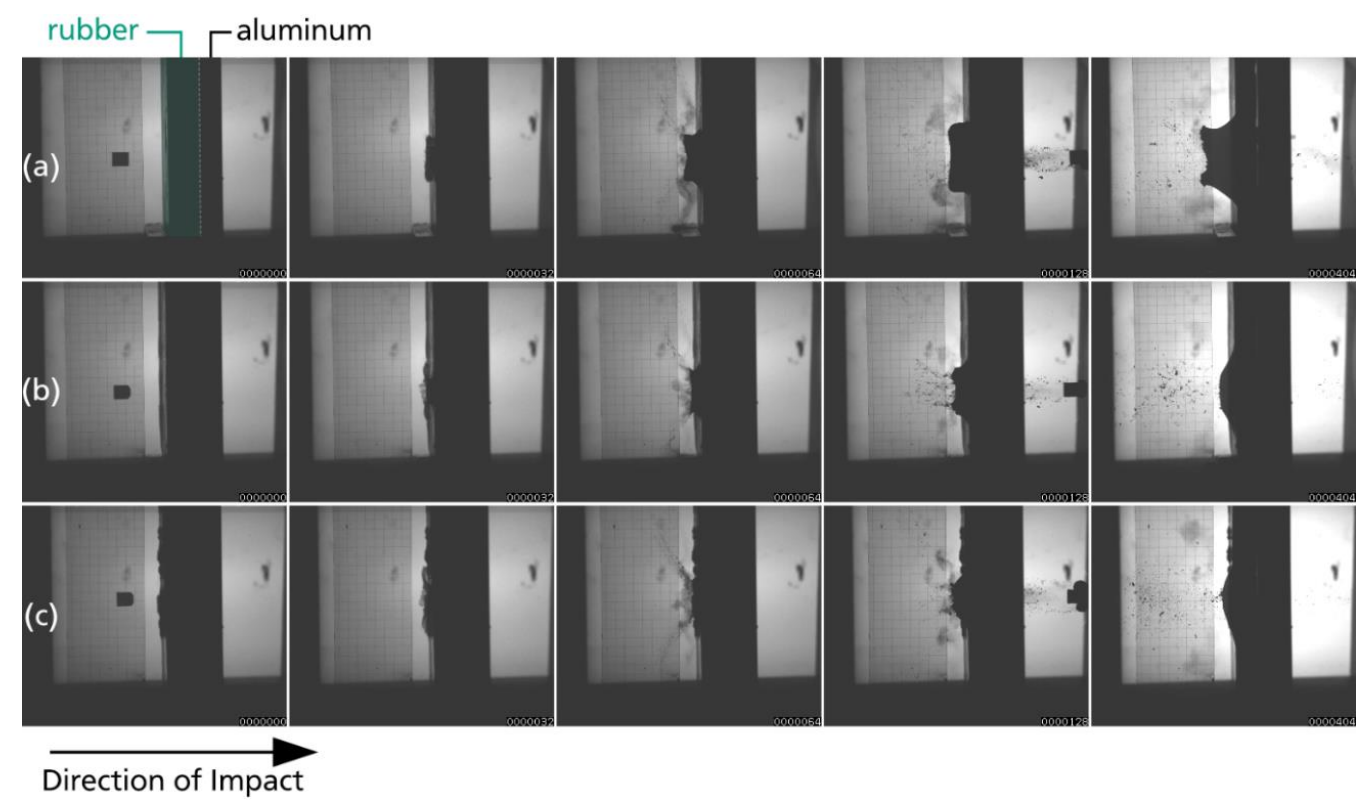

Figure 8. High-speed photographs of the impact behavior of (a) BR/NR (9:1), (b) IIR, (c) SBR. Photographs were taken after 0, 32, 64, 128 and $404 \mu \mathrm{s}$. A schematic colorized overlay in the first photograph (a) highlights the rubber front-layer and aluminum back-layer.

The impact responses of the materials with different glass transition temperatures vary strongly. BR/NR (Figure 8a) shows a ductile behavior whereas the rubber bulges away from the projectile. SBR (Figure 8c) responds in a brittle manner and the rubber splinters away. This behavior is expected 
since the glass transition temperature of SBR is too high, meaning the impact frequency is higher than the glass transition frequency, and thus the material undergoes the transition while the glass transition temperature of $\mathrm{BR} / \mathrm{NR}$ is too low, leading to the impact not triggering the glass transition.

For the IIR specimen (Figure 8b), the material shows a mix of both responses. The rubber splintered away like in the case of SBR, but bulging can also be observed. This can be interpreted as the glass transition being in the correct magnitude. As the mean molecular mass of elastomers and polymers, in general, has a broad distribution, its properties also show a larger variety. Some of the polymer chains already underwent the phase transition from ductile to brittle, others still behave in a ductile manner.

The results of the DMA experiments in Section 3.2, stating that impact frequencies are in the range of the glass transition of IIR, can be validated by these impact behavior experiments. By using an ultra-high-speed camera, it is clearly possible to differentiate between IIR and other rubbers with non-optimized glass transition temperatures.

\subsection{Energy of Absorption of Armor Plates}

To provide another proof of the proposed rubber compounds, the same rubber formulations were examined as part of steel-rubber-aluminum sandwich plates. Using flash X-ray images, the residual velocity $v_{r}$ of the projectile was determined. The kinetic energy $E_{k i n}$ before and after the impact can be calculated using

$$
E_{k i n}=1 / 2 \cdot m \cdot v 2,
$$

with $m$ being the mass of the projectile $\left(4.6 \mathrm{~g}\right.$ ) and $v$ being either the impact velocity $v_{0}$ or the residual velocity $v_{r}$. The absorption parameter $A$ describes the percentual amount of absorbed kinetic energy and can be calculated as follows:

$$
A=\frac{E_{k i n, r}}{E_{k i n, 0}}
$$

The results show that the kinetic energy absorption is highly dependent on the impact velocity $v_{0}$. To compare the obtained results, it is necessary to either limit the results used to shots where the impact velocity $v_{0}$ is in a narrow range around a specific velocity (e.g., $600 \mathrm{~m} / \mathrm{s}$ ) or to take into account the standardization of the absorption values. The dependency between the absorption parameter $A$ and the impact velocity $v_{0}$ for the different armor configurations can be seen in Figure 9 . As a reference, the same configuration without the rubber interlayer ( $6 \mathrm{~mm}$ steel $+4 \mathrm{~mm}$ aluminum) is used.

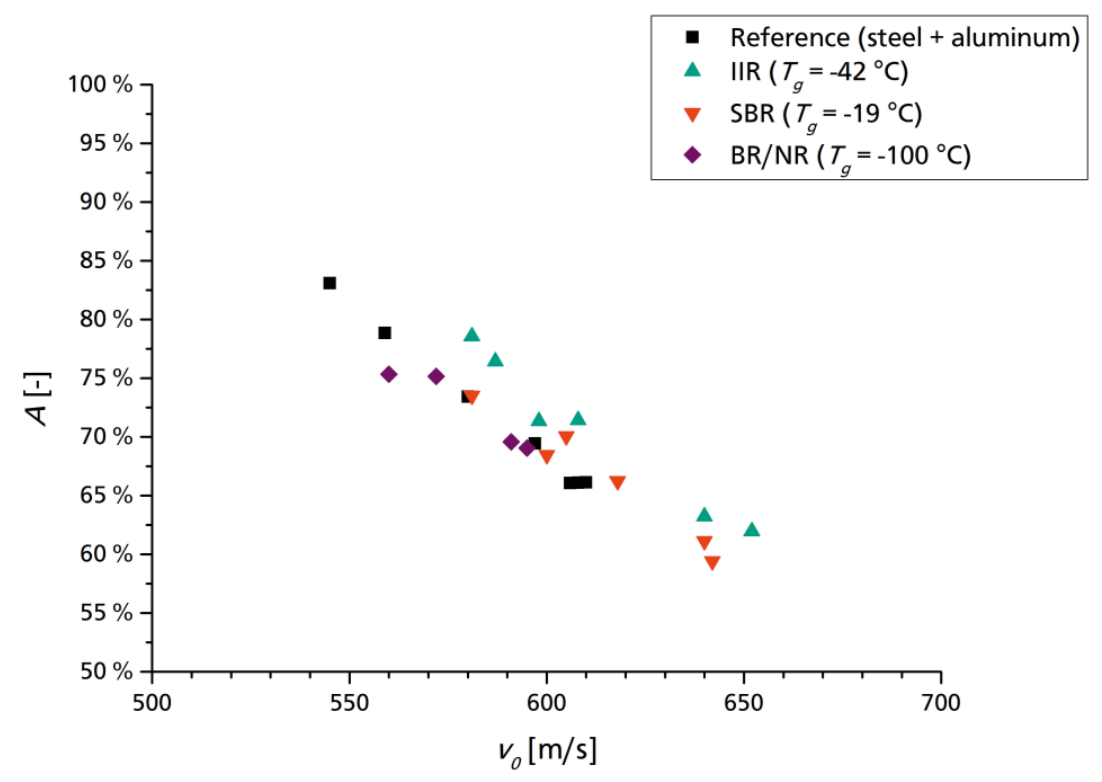

Figure 9. Dependency between absorption parameter $A$ and impact velocity $v_{0}$ for different armor configurations. 
At every impact velocity $v_{0}$, butyl rubber shows better energy absorption than the other configurations. To better compare the actual energy absorption, the values of the absorption parameter $A$ are standardized to an impact velocity $v_{0}$ of $600 \mathrm{~m} / \mathrm{s}$ according to

$$
A_{600}=A \cdot \frac{600 \mathrm{~m} / \mathrm{s}}{v_{0}} .
$$

The standardized mean values for each configuration are given in Table 5 and plotted in Figure 10. $A_{600} / A_{600, \text { ref }}$ is used as a measurement for the improvement over the reference configuration without rubber.

Table 5. Mean values of energy absorption $A$ with a standardized impact velocity $v_{0}$ of $600 \mathrm{~m} / \mathrm{s}$ for the investigated configurations with different rubbers.

\begin{tabular}{cccc}
\hline Specimen & $T_{g}\left[{ }^{\circ} \mathrm{C}\right]$ & $A_{600}[-]$ & $A_{600} / A_{600, r e f}[-]$ \\
\hline Reference (without rubber) & - & $68.0 \pm 1.5 \%$ & $0.0 \%$ \\
SBR & -19 & $73.6 \pm 1.8 \%$ & $2.4 \%$ \\
IIR & -42 & $69.6 \pm 1.3 \%$ & $8.3 \%$ \\
BR/NR & -100 & $69.8 \pm 1.3 \%$ & $2.6 \%$ \\
\hline
\end{tabular}

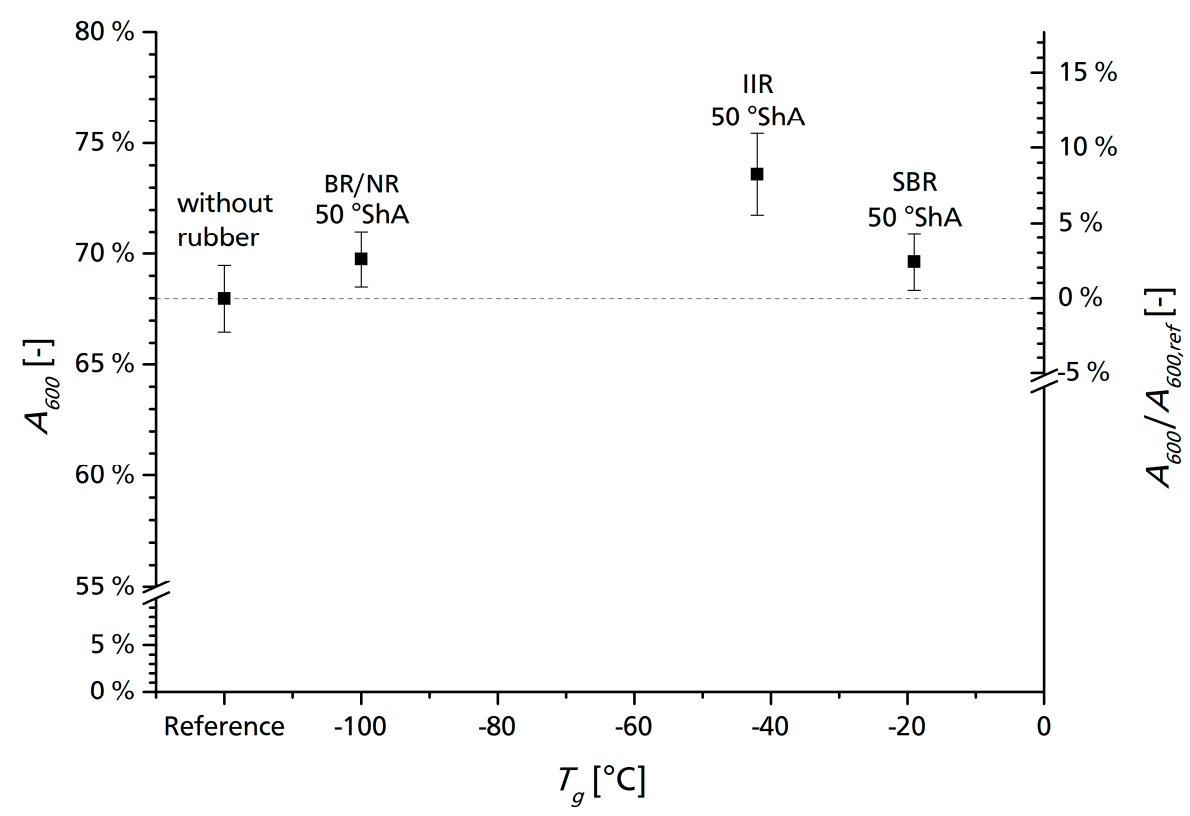

Figure 10. Energy absorption during ballistic impact experiments for the configurations with different rubbers. All three rubber compounds possess the same hardness to decrease potential influences besides glass transition and maximized damping.

While all rubbers show at least a small improvement over the reference configuration, butyl rubber shows a significant increase of the energy absorption of $8.3 \%$.

Another set of ballistic experiments was carried out to investigate the influence of both hardness and damping of the rubber layer on ballistic protection, in addition to the previously analyzed influence of the glass transition temperature $T_{g}$. Experiments were carried out with the rubber compound showing the most promising results in the previous experiment (IIR). In principle, the same formulation for IIR like in the previous experiments was used, but hardness and damping were varied by changing the carbon black content of the formulation. It has to be noted that it is not possible to vary both properties individually, as the carbon black content (or any other formulation parameter for that matter) will always have an influence on them simultaneously. With an increase in carbon black content the hardness will increase, and the damping $(\tan \delta)$ will decrease. The standardized mean values for each 
configuration are given in Table 6 and plotted in Figure 11. The IIR compound with 50 phr carbon black and a hardness of $50^{\circ} \mathrm{ShA}$ is the same rubber compound that was used for the experiments shown in Figure 10.

Table 6. Mean values of energy absorption $A$ with a standardized impact velocity $v_{0}$ of $600 \mathrm{~m} / \mathrm{s}$ for the investigated configurations using IIR formulations with varying carbon black (CB) content.

\begin{tabular}{ccccc}
\hline Specimen & $\operatorname{ShA}\left[{ }^{\circ} \mathbf{S h A}\right]$ & $\tan \delta[-]$ & $A_{\mathbf{6 0 0}}[-]$ & $A_{\mathbf{6 0 0}} / A_{\mathbf{6 0 0}, \text { ref }}[-]$ \\
\hline $\begin{array}{c}\text { Reference (without } \\
\text { rubber) }\end{array}$ & - & - & $68.0 \pm 1.5 \%$ & $0.0 \%$ \\
IIR, 20 phr CB & 36 & 1.26 & $67.9 \pm 2.0 \%$ & $0.0 \%$ \\
IIR, 35 phr CB & 42 & 1.06 & $69.3 \pm 1.0 \%$ & $1.9 \%$ \\
IIR, 50 phr CB & 50 & 0.83 & $73.6 \pm 1.8 \%$ & $8.3 \%$ \\
IIR, 65 phr CB & 57 & 0.64 & $73.5 \pm 2.1 \%$ & $8.1 \%$ \\
IIR, 80 phr CB & 64 & 0.41 & $71.4 \pm 2.0 \%$ & $5.1 \%$ \\
\hline
\end{tabular}

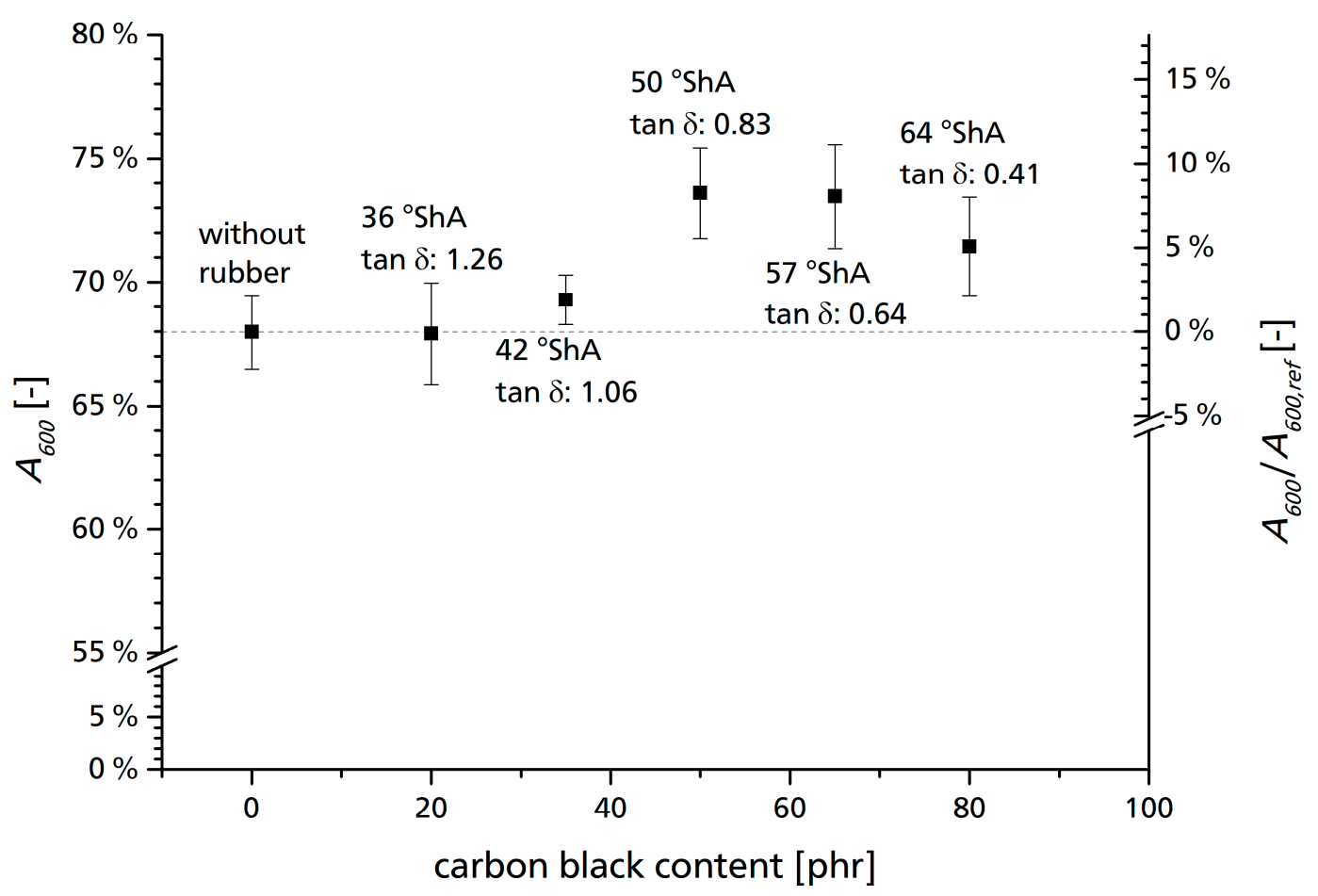

Figure 11. Energy absorption during ballistic impact experiments for configurations using IIR formulations with varying carbon black content.

It is assumed that both high hardness and high damping of the rubber have a positive impact the effectiveness of the armor configuration. As both properties are conversely dependent on the varied carbon black content, it is expected that there is an overlapping of both effects. In that case, the best energy absorption should be observed for intermediate carbon black contents. As can be seen in the results, there seems to be a maximum in energy absorption around the configurations with a carbon black content of 50 and 65 phr. For very low hardnesses, the effectiveness of the armor is not better than the reference, while for a very high hardness but a low damping, the energy absorption gets smaller again.

From these results it can be concluded that while both hardness and damping of the rubber interlayer have an influence on ballistic protection, the influence of hardness is more important and the best results are achieved with medium carbon black contents of around 50-65 phr. It might be possible to further increase the energy absorption by finding the optimum in carbon black content, but given the small overall improvement, it is not expected that this will yield much better results. 


\section{Discussion}

Following the assumption that by optimizing material properties, a ballistic impact can trigger the glass transition, rubber should significantly add to the ballistic protection of multilayered armor. In this case, the damping of the rubber is maximized in the area of the glass transition. Using dynamic mechanical analysis and the WLF equation, it is shown that this is the case for the self-developed butyl rubber (IIR). Ballistic tests were applied for further evaluation of the assumption. By observing the behavior of a rubber-coated aluminum plate under a high-velocity impact, it can be seen that this material shows an improved behavior compared to other rubbers with non-optimized glass transition temperatures, like styrene-butadiene rubber (SBR) or butadiene rubber/natural rubber (BR/NR). In consequence, the conclusion can be stated that this butyl rubber is in the glass transition zone during the impact, which affects the behavior of the rubber itself and improves the performance of the total armored laminate.

To further validate that this phenomenon not only occurs but also increases the protective effectiveness during ballistic impacts, ballistic tests were carried out to determine the energy absorption of multilayered steel-rubber-aluminum armors. Different rubbers (IIR, SBR and BR/NR) are compared to each other and a reference configuration without a rubber interlayer. The configuration with butyl rubber as the interlayer shows better results with an increase compared to the reference configuration of around $8 \%$, while the rubbers with the non-optimized glass transition temperature only reach an increase between $2 \%$ and $3 \%$.

Additionally, the influence of carbon black content and thus the influence of hardness and damping were investigated. It can be concluded that both material properties play a role in determining the effectiveness of the armor, while hardness shows the bigger influence. An optimum can be reached with intermediate carbon black contents of around 50-65 phr. Linking both series of ballistics, this means that even if the maximum energy absorption is not reached because of using not enough carbon black and thus having a too low hardness, IIR is still is on the same level as a worse-suited compound like $\mathrm{BR} / \mathrm{BR}$, even if the latter possesses a higher hardness.

It is necessary to emphasize that every possible weight reduction is positive and an important input to armor development. According to VPAM [20], to achieve VPAM 10 level, RHA steel with a thickness of $18 \mathrm{~mm}$ is necessary to provide protection from small caliber projectiles. With a density of steel of $7.8 \mathrm{~g} / \mathrm{cm}^{3}$ that correlates to an areal density of $140 \mathrm{~kg} / \mathrm{m}^{2}$. Assuming that the surface of an average car is around 8-12 $\mathrm{m}^{2}$ [23], this would mean that only the ballistic armor of an armored vehicle would weigh around $1100-1700 \mathrm{~kg}$. An $8 \%$ decrease of the total weight achieved by using an optimized armor configuration would reduce the total vehicle weight by over $100 \mathrm{~kg}$.

\section{Conclusions}

In an attempt to increase the effectiveness of rubber interlayers in sandwich-armors, the research focus was put on optimizing viscoelastic properties. By making use of the principle of time-temperature superposition, the ideal glass transition temperature for the given impact frequency range was calculated. With this knowledge, a butyl rubber compound with its glass transition temperature $T_{g}$ in the ideal range was manufactured. By using ballistic experiments, it was compared to other rubber compounds (BR/NR, SBR) that feature glass transition temperatures outside of the ideal range. The experimental results support the stated claim that ballistic protection is positively influenced by having the rubber layer undergo its glass transition during impact and thus maximize its damping.

The influence of hardness and damping of the rubber compound was further analyzed by varying the carbon black content of the rubber formulation, resulting in different values for hardness and damping. The medium carbon black content, and thus medium hardness and damping, showed the best results for the application with an increase of energy absorption by $8 \%$ over a reference without the rubber interlayer. 
Author Contributions: Conceptualization: J.K., L.P. and T.F.; methodology, J.K. and F.K., software, F.K. and N.F.; validation, J.K.; formal analysis, J.K., F.K. and N.F.; investigation, J.K., F.K. and N.F.; resources, L.P. and T.F.; data curation, J.K., F.K. and N.F.; writing-original draft preparation, J.K.; writing-review and editing, J.K.; visualization, J.K.; supervision, L.P. and T.F.; project administration, T.F.; funding acquisition, T.F. All authors have read and agreed to the published version of the manuscript.

Funding: This research received no external funding.

Acknowledgments: The authors would like to thank Safic Alcan, Hansen and Rosenthal, and Arlanxeo for providing free samples of the materials used.

Conflicts of Interest: The authors declare no conflict of interest.

\section{Abbreviation/Symbol}

$\begin{array}{ll}A & \text { Absorption parameter } \\ \alpha_{V} & \text { Thermal expansion coefficient } \\ \text { AP } & \text { Armor piercing } \\ \beta_{T} & \text { Frequency shift factor } \\ \text { BR } & \text { Butadiene Rubber } \\ C_{1}, C_{2} & \text { Empirical material and reference temperature } \\ \text { CB } & \text { dependent parameters } \\ d & \text { Carbon black } \\ \text { DMA } & \text { Armor thickness } \\ E^{\prime} & \text { Dynamic mechanical analysis } \\ E^{\prime \prime} & \text { Storage modulus } \\ E_{k i n} & \text { Loss modulus } \\ f_{g} & \text { Kinetic energy } \\ f_{i m p a c t} & \text { Glass transition frequency } \\ \text { FSP } & \text { Impact frequency } \\ \text { IIR } & \text { Fragment simulating projectile } \\ m & \text { Butyl rubber } \\ \text { MBTS } & \text { Mass of projectile } \\ \text { NR } & \text { Mercaptobenzothiazole disulfide } \\ \text { phr } & \text { Natural rubber } \\ \text { RHA } & \text { Parts per hundred rubber } \\ \text { SBR } & \text { Rolled homogenous armor } \\ \text { ShA } & \text { Styrene-butadiene rubber } \\ T & \text { Shore A hardness } \\ t & \text { Temperature } \\ T_{0} & \text { Time } \\ \text { tan } \delta & \text { Reference temperature } \\ T_{g} & \text { Loss factor } \\ \text { TMTD } & \text { Glass transition temperature } \\ v & \text { Tetramethylenethiuram disulfide } \\ v_{0} & \text { Velocity } \\ v_{50} & \text { Projectile velocity } \\ \text { VPAM } & \text { Ballistic limit } \\ v_{r} & \text { Vereinigung der Prüfstellen für angriffshemmende } \\ \text { WLF } & \text { Residual velocity } \\ & \end{array}$

\section{References}

1. Madhu, V.; Bhat, T.B. Armour Protection and Affordable Protection for Futuristic Combat Vehicles. Def. Sci. J. 2011, 61, 394-402. [CrossRef]

2. Hazell, P.J. Armour-Materials, Theory and Design; CRC Press: Boca Raton, FL, USA, 2016. 
3. Crouch, I.G. The Science of Armour Materials; Woodhead Publishing: Cambridge, UK, 2016.

4. Yang, Y.; Chen, X. Energy absorption among layers in the multiply system under ballistic impact. In Proceedings of the 89th Textile Institute World Conference, Wuhan, China, 2-6 November 2014.

5. Gamache, R.M.; Giller, C.B.; Montella, G.; Fragiadakis, D.; Roland, C.M. Elastomer-metal laminate armor. Mater. Des. 2016, 111, 362-368. [CrossRef]

6. Hunkler, R. Untersuchungen zur Wechselwirkung von Stahl-Gummi-Stahl-Sandwiches mit KE-Penetratoren; Deutsch-Französisches Forschungsinstitut Saint-Louis: Saint-Louis, France, 1993.

7. Fras, T.; Fras, L.J.; Faderl, N. Rubber and magnetorheological fluid applied as the interlayer in composite armours against high-velocity loadings. Diagnostyka 2017, 18, 63-68.

8. Fras, T.; Karl, J.; Faderl, N. Protection against Small Calibers; Deutsch-Französisches Forschungsinstitut Saint-Louis: Saint-Louis, France, 2018.

9. Roland, C.M.; Fragiadakis, D.; Gamache, R.M. Elastomer-Steel Laminate Armor. In 2010 NRL Review: Power, Energy, Synergy; Naval Research Laboratory: Washington, DC, USA, 2010; pp. 148-154.

10. Röthemeyer, F.; Sommer, F. Kautschuktechnologie; Carl Hansler Verlag: Munich, Germany, 2013.

11. Tieke, B. Makromolekulare Chemie; Wiley-VCH: Hoboken, NJ, USA, 2005.

12. Fragiadakis, D.; Gamache, R.; Bogoslovov, R.B.; Roland, C.M. Segmental dynamics of polyurea: Effect of stoichiometry. Polymer 2010, 51, 178-184. [CrossRef]

13. Bogoslovov, R.B.; Roland, C.M.; Gamache, R.M. Impact-induced glass transition in elastomeric coatings. Appl. Phys. Lett. 2007, 90, 221910. [CrossRef]

14. SSAB. Armox 370T. Available online: https://www.ssab.com/products/brands/armox/armox-370t-class-2 (accessed on 4 August 2020).

15. Aalco. Al 5754. Available online: http://www.aalco.co.uk/datasheets/Aluminium-Alloy-5754-H111Treadplate_142.ashx (accessed on 4 August 2020).

16. Faderl, N. Moderne Mess- und Visualisierungsverfahren in der Endballistik. In Proceedings of the Endballistik-Grundlagen und Anwendungen, Saint-Louis, France, 27-29 June 2017.

17. NATO Standardization Agency. Ballistic Test Method for Personal Armour Materials and Combat Clothing, 2nd ed.; STANAG 2920 PPS; NATO Standardization Agency: Brussels, Belgium, 2003.

18. Williams, M.L.; Landel, R.F.; Ferry, J.D. The Temperature Dependence of Relaxation Mechanisms in Amorhpous Polymers and Other Glass-forming Liquids. J. Am. Chem. Soc. 1955, 77, 3701-3707. [CrossRef]

19. Yildiz, M.E.; Kokini, J.L. Determination of Williams-Landel-Ferry constants for a food polymer system: Effect of water activity and moisture content. J. Rheol. 2001, 45, 903-912. [CrossRef]

20. VPAM. Allgemeine Richtlinie für Ballistische Material- Konstruktions- und Produktprüfungen; VPAM: Dordrecht, The Netherlands, 2006.

21. Capps, R.N. Elastomeric Materials for Acoustical Applications; Naval Research Laboratory: Washington, DC, USA, 1989.

22. Frankovich, D. The Basics of Vibration Isolation Using Elastomeric Materials; Aearo Technologies: Indianapolis, IN, USA, 2009.

23. Secondskinaudio. Square Footage Help. Available online: https://www.secondskinaudio.com/squarefootage-help/ (accessed on 4 August 2020).

Publisher's Note: MDPI stays neutral with regard to jurisdictional claims in published maps and institutional affiliations.

(C) 2020 by the authors. Licensee MDPI, Basel, Switzerland. This article is an open access article distributed under the terms and conditions of the Creative Commons Attribution (CC BY) license (http://creativecommons.org/licenses/by/4.0/). 